DOI: https://doi.org/10.11144/Javeriana.upsy17-1.eerp

\title{
Efectos de la estructura de las redes personales en la red sociocéntrica de una cohorte de estudiantes en transición de la enseñanza secundaria a la universidad*
}

Effects of the Structure of Personal Networks in the Acquaintances Network of a Cohort of Students in Transition from High School to University

Recepción: 01 Septiembre 2015 | Aprobación: 06 Junio 2017

\author{
ISIDRO MAYA JARIEGO \\ Universidad de Sevilla, España \\ ORCID: http://orcid.org/0000-0002-2568-5048 \\ Daniel Holgado Ramos \\ Universidad de Sevilla, España \\ Miranda J. LubBers \\ Universidad Autónoma de Barcelona, España
}

a Autor de correspondencia. Correo electrónico: isidromj@us.es

Para citar este artículo: Maya Jariego, I., Holgado, D., \& Lubbers, M. (2018). Efectos de la estructura de las redes personales en la red sociocéntrica de una cohorte de estudiantes en transición de la enseñanza secundaria a la universidad. Universitas Psychologica, 17(1), 1-12. https://doi.org/10.11144/Javeriana.upsy 17-1.eerp

\section{RESUMEN}

En este trabajo se examina la transición normativa de una cohorte de estudiantes de Enseñanza Secundaria de Alcalá de Guadaíra, que empiezan sus estudios universitarios en Sevilla. Se analiza la evolución de la red social de la cohorte de estudiantes junto con los cambios que experimentan en la composición y la estructura de sus redes personales. El seguimiento longitudinal se desarrolla a lo largo de 17 meses en los que los encuestados $(n=57)$ empiezan a distribuir su tiempo entre la localidad en la que residen y la ciudad en la que estudian, de modo que en su conjunto aumentan la frecuencia de los viajes de ida y vuelta a la capital. Este es uno de los primeros estudios que recoge información de las redes personales de los miembros de una red social completa, combinando ambos indicadores en los análisis. Con el modelo de simulación longitudinal de redes sociales, utilizando el software RSIENA, comprobamos que la intermediación promedio de las redes personales tiene un efecto significativo en la evolución de la red social de los estudiantes que terminan la Enseñanza Secundaria. La intermediación promedio también parece incidir en la tasa de cambio del sentido de comunidad con la ciudad de residencia. Concluimos proponiendo que el desacoplamiento de las relaciones, junto con la competencia entre relaciones múltiples activas, constituyen dos mecanismos clave de los cambios en la estructura de las redes personales en proceso de transición ecológica.

Palabras clave

transiciones ecológicas; movilidad metropolitana; redes personales; redes sociales; simulación longitudinal de redes sociales; RSIENA.

\section{ABSTRACT}

In this work the normative transition of a cohort of secondary school students from Alcalá de Guadaíra that start college education in Seville is examined. The evolution of the social network of the cohort of students 
together with the changes experienced in the composition and structure of their personal networks is analyzed. The longitudinal tracking is developed over 17 months when respondents $(n=57)$ begin to distribute their time between the town in which they live and the city where they study, so there is an increase in the frequency of commuting to the capital. This is one of the first studies collecting information from the personal networks of the members of a complete social network, and combining both personal and sociocentric indicators in the analysis. With models for the longitudinal analysis of social networks, using the RSIENA software, we found that average betweenness of personal networks has a significant effect on the social network of students completing secondary education. The average betweenness also seems to influence the change rate in the sense of community with the city of residence. We conclude by proposing that the decoupling of relations, as well as active competition between multiple relationships, are two key mechanisms of changes in the structure of personal networks in the process of ecological transition.

Keywords

ecological transitions; commuting; personal networks; social networks; social networks longitudinal simulation; RSIENA.

Una transición ecológica se produce cuando la posición de una persona en el ambiente se ve modificada como resultado de un cambio de rol, de contexto o ambos (Bronfenbrenner, 1977). Dichas transiciones representan un momento de cambio en el patrón de relaciones entre el individuo y el contexto. En consecuencia, se trata de puntos de inflexión que conllevan un riesgo o una oportunidad.

La diferenciación más extendida distingue entre las transiciones normativas y las nonormativas (Arnett, 2000; Connell \& Furman, 1984; Ruble \& Seidman, 1996). Las primeras le ocurren al conjunto de la población en determinados periodos evolutivos y/o a determinadas edades, o bien están vinculadas a un itinerario planificado institucionalmente. Por ejemplo, las transiciones académicas, tales como terminar la enseñanza primaria o la enseñanza secundaria, son realizadas a menudo por grandes cohortes de individuos y están previstas de antemano. Por el contrario, las transiciones nonormativas no le ocurren a todo el mundo, y suceden de modo irregular. Por ejemplo, la inmigración internacional es un tipo de cambio que vive una parte de la población, pero no otra, y que discurre irregularmente de acuerdo con el ciclo vital y con las circunstancias socioeconómicas.

Tales procesos pueden tener una tasa de cambio diferente en los niveles colectivo e individual, como lo establecieron Seidman y French (2004), quienes analizaron la confluencia de las trayectorias evolutivas con las transiciones ecológicas normativas en un estudio longitudinal con adolescentes urbanos de bajos ingresos. Cuando los estudiantes cambian de escuela, al pasar de la enseñanza primaria a la enseñanza secundaria, se transforma el conjunto de sus relaciones de rol, con los compañeros de escuela, con los profesores y con la familia. En ese caso, la transición coincide con un período evolutivo crítico. Pese a que la acumulación de cambios constituye un contexto de riesgo, el acompañamiento por parte de un grupo de iguales -que está expuesto a las mismas circunstancias- puede tener un valor preventivo y, bajo determinadas condiciones, convertirse en un contexto de oportunidades y crecimiento personal. Vivir la transición como parte de un colectivo parece demorar ligeramente los cambios que se experimentan en el plano personal.

En este trabajo partimos del supuesto de que los cambios en las redes personales reflejan indirectamente la transición ecológica que experimentan los individuos (Maya Jariego, 1999, 2006). En estudios anteriores, hemos evaluado la estructura y la composición de las redes personales de individuos en situación de movilidad geográfica para describir las transformaciones psicológicas individuales. Con una muestra representativa de una ciudad de tamaño medio en el entorno metropolitano de Sevilla, comprobamos una relación inversa entre la frecuencia de los desplazamientos metropolitanos y el grado de cohesión estructural de las redes personales (Maya Jariego \& Holgado, 2015). De hecho, en una encuesta transversal con estudiantes universitarios de la misma ciudad -un colectivo que se caracteriza por ser más móvil que el resto de la población-, obtuvimos puntuaciones en la centralidad 
promedio comparativamente más bajas, con la excepción del indicador de intermediación (average betweenness), que era más elevado en la muestra supuestamente más móvil (Araya \& Maya Jariego, 2005; Maya Jariego \& Holgado, 2005).

La red personal proporciona información subjetiva sobre el espacio de relaciones interpersonales de un individuo. La red social completa, en cambio, es de carácter intersubjetivo y analiza la interacción social entre un conjunto de individuos en un espacio determinado. Tanto los indicadores analíticos de centralidad y posición, como el estudio de las agrupaciones y las propiedades estructurales, se desarrollaron casi en su integridad en la tradición de las redes completas. Recientemente, el enfoque estructural empezó a aplicarse también en el estudio de las redes personales (McCarty, 2002). En este contexto, apenas hay investigaciones, como proponemos en este artículo, que hayan examinado la co-variación o la co-determinación de las redes personales y las redes completas. A continuación, exploramos el papel de las redes personales en la evolución de las relaciones en una cohorte de estudiantes que termina sus estudios de enseñanza secundaria $y$, en una proporción significativa, inicia sus estudios universitarios.

Por tanto, con una encuesta longitudinal, en este trabajo empírico combinamos dos innovaciones, puesto que evaluamos las transiciones ecológicas (a) con indicadores estructurales de redes, y (b) en los niveles individual y colectivo simultáneamente.

\section{Método}

\section{Participantes}

En un estudio longitudinal, recabamos información de la red social de 69 estudiantes del último curso del Instituto Albero en Alcalá de Guadaíra, una ciudad del entorno metropolitano de Sevilla, a unos 16 kilómetros de distancia de la capital. La segunda oleada se realizó 17 meses después de la primera entrevista, con un total de 57 encuestados. En la primera oleada, completaron la encuesta 73 personas de una lista de 77 estudiantes de último curso (94.8\%). Cuatro entrevistas se descartaron por disponer de información incompleta. En el seguimiento, se consiguió encuestar a 57 de los 69 encuestados finales de la primera oleada, con lo que se alcanzó al $82.6 \%$ del total. La primera oleada estuvo compuesta por 31 hombres (44.9\%) y 38 mujeres $(55.1 \%)$ que viven en Alcalá de Guadaíra, y que en su mayoría tienen la expectativa de vivir en Alcalá (36.2 \%) o en Sevilla (37.7\%) en el futuro. En la segunda oleada hay 27 hombres $(47.4 \%)$ y 30 mujeres $(52.6 \%)$.

\section{Instrumentos}

En ambas oleadas se recogió información sobre la red social de amigos y conocidos en la cohorte de estudiantes analizada, las redes personales individuales, información sobre los patrones de movilidad metropolitana, datos de la distribución del tiempo entre localidades e información demográfica de carácter complementario (con $n$ $=69$ en la primera oleada y $n=57$ en la segunda, en todos los casos).

\section{Red social}

Cada encuestado revisó el listado de los 77 matriculados en el último curso del IES Albero, en donde se indicó en cada caso, las relaciones que mantenían con cada uno de ellos. El listado es un procedimiento exhaustivo de recogida de información que permite obtener una lista más amplia de relaciones, incluyendo los lazos más débiles. $\mathrm{Al}$ respecto de cada nombre, los encuestados indicaban el tipo de relación: (1) "me suena su nombre", (2) "lo conozco", (3) "hablamos de vez en cuando", (4) "tenemos bastante relación" o (5) "somos amigos". Aunque en otros análisis hemos trabajado con relaciones valoradas (Maya Jariego, Lubbers, \& Molina, 2015), en este caso dicotomizamos la red de dos maneras para analizar la red de conocidos $(\geq 2)$ y la red de lazos fuertes $(\geq 4)$. Finalmente, la red 
de conocidos se basa en 3969 lazos observados en T1 $(n=69)$ y 2796 en T2 $(n=57)$.

\section{Redes personales}

Se siguió la metodología de evaluación de la estructura de las redes personales propuesta por McCarty (2002), estableciendo un número fijo de alteri (o lista de contactos interpersonales habituales). En este caso, se generó una lista de 45 alteri y sus relaciones mutuas en cada entrevista. Como generador de nombres, se utilizó la entrevista semi-estructurada de apoyo social de Arizona (ASSIS), diseñada originalmente por Barrera (1980). Una vez obtenida la primera lista de nombres, que oscilaba entre 10 y 15 alteri aproximadamente $(M=12.55, D T=4.59)$, se les pidió que completaran el listado hasta obtener 45 nombres en total, y que mencionaran personas con las que mantenían relación habitual. Sobre cada nombre, los encuestados indicaban el lugar de residencia (Alcalá, Sevilla u otra ciudad) y el tipo de relación (distinguiendo entre parientes, amigos, conocidos o compañeros de curso, vecinos y "otros").

Una definición básica de los indicadores de centralidad utilizados en este artículo puede consultarse en McCarty (2002). Por nuestra parte, prestaremos especial atención al indicador de intermediación (betweenness). La centralidad de intermediación nodal es el número de veces que un nodo hace de puente entre los caminos más cortos entre pares de actores (Freeman, 1977). Los nodos que aparecen con frecuencia entre caminos geodésicos tienen una alta intermediación, y suelen integrar módulos diferenciados de la red. Como indicador de resumen, utilizamos el promedio de los indicadores individuales de intermediación (average betweenness), que informa de manera genérica sobre el grado de modularidad de la red personal.
Movilidad metropolitana y aspectos sociodemográficos

La encuesta se completaba con información demográfica, con especial atención a aspectos relacionados con la movilidad geográfica. Los encuestados indicaron la frecuencia de desplazamientos entre Alcalá y Sevilla, y eligieron entre "menos de una vez al mes", "una vez al mes", "una vez a la semana", "cinco veces a la semana" y "una vez al día". También se les pidió que indicaran, tomando como referencia una semana ordinaria, cómo distribuían un $100 \%$ teórico del tiempo semanal entre ambas ciudades.

\section{Sentido de comunidad}

La interacción vecinal y el arraigo territorial son dinámicas básicas de la integración urbana (Gómez-Jacinto \& Hombrados-Mendieta, 1992; Sánchez-Vidal, 2001; Valera, 1997). En este estudio, aplicamos el Sense of Community Index de 12 ítems (Chavis, Hogge, McMillan, \& Wandersman, 1986), que en estudios anteriores hemos utilizado con inmigrantes internacionales (Domínguez \& Maya Jariego, 2008) y con población en situación de movilidad metropolitana (Maya Jariego \& Armitage, 2007). Como ocurre con la original, la versión española de la escala permite evaluar los factores de pertenencia, influencia, satisfacción de necesidades y conexión emocional compartida (Maya Jariego, 2004; McMillan \& Chavis, 1986). En este caso, se administró por duplicado, referido a la localidad de residencia (Alcalá de Guadaíra) y a la capital en la que normalmente cursan sus estudios universitarios (Sevilla). Se obtuvo una fiabilidad de 0.572 (T1) y 0.54 (T2) en la versión referida a Alcalá; y de 0.574 (T1) y 0.508 (T2) en la de Sevilla. Son indicadores que están solo ligeramente por debajo del rango habitual con este instrumento, en parte por contar con una muestra relativamente reducida. 
Análisis de datos

Las 57 matrices de redes personales fueron tratadas y analizadas con Ucinet 6 (Borgatti, Everett, \& Freeman, 2002). Las matrices de redes completas en las dos oleadas de observación también fueron tratadas con Ucinet 6 para derivar indicadores descriptivos, y a continuación, fueron representadas gráficamente con Visone 2.8 (Brandes \& Wagner, 2004). Tanto los datos de encuesta como los indicadores de redes personales y redes sociales obtenidos previamente, fueron analizados con SPSS 22. Finalmente, el análisis longitudinal de las redes sociales se llevó a cabo con el programa RSIENA (Snijders, 2001, 2005). RSIENA se basa en un modelo de probabilidad multinomial para la creación y la disolución de relaciones sociales dentro de un grupo de actores. Concretamente, simula la evolución de una red completa a partir de la primera observación de la red, basándose en un modelo teórico de cambio. El modelo teórico está compuesto por diferentes funciones. Por un lado, la función de la tasa de cambio especifica qué actores son más propensos a cambiar sus relaciones. En segundo lugar, la función de evaluación especifica qué relaciones son más propensas a cambiar, dependiendo de cada actor específico. Ambas funciones son similares a las funciones de regresión, e incluyen efectos y parámetros. Los efectos pueden ser estructurales o relacionados con los atributos de los actores. Los parámetros se estiman comparando un proceso de simulación iterativa con las observaciones consecutivas de la red. El modelo también permite el análisis de la mutua dependencia de la evolución de la red y del comportamiento de los actores (Snijders, 2001, 2005; para una introducción en castellano, véase De Federico, 2005; Maya Jariego \& De Federico, 2006).

\section{Resultados}

\section{Entrando en un estilo de vida metropolitano}

En apenas un año y medio, la cohorte de estudiantes encuestada experimenta cambios significativos en su vida, tanto a nivel individual como colectivo. Como punto de partida, se trata de un grupo homogéneo de jóvenes de 17 años $(M=17.2, D T=0.66)$, que viven con sus padres y que han vivido toda su vida en la misma ciudad. Diecisiete meses después de la primera entrevista, más de la mitad de ellos ha iniciado sus estudios universitarios (56.4\%), mayoritariamente en la Universidad de Sevilla o en la Universidad Pablo de Olavide. En consecuencia, es habitual que visiten con más frecuencia la capital y pasen comparativamente más tiempo en Sevilla.

En la primera oleada, el grueso de los encuestados viaja menos de una vez por semana a Sevilla (91.3\%), mientras que en la segunda observación más de la mitad viaja cinco o más veces por semana a la capital $(56.5 \%)$. En su conjunto, pasan de estar casi todo el tiempo en su localidad de residencia (86.9\%) a distribuirlo entre Alcalá (63.4\%) y Sevilla (36.6\%).

Estos cambios discurren en paralelo a otras transformaciones en la identificación comunitaria de los estudiantes. En la primera entrevista, se identifican más con Alcalá ( $M$ $=31.74, \mathrm{DT}=4.88)$ que con Sevilla $(\mathrm{M}=$ 27.31, DT $=4.75)(t=-5.261, p<0.0001)$. Lo mismo se observa en la segunda oleada. La diferencia entre Alcalá $(M=29.69$, DT $=$ 4.72) y Sevilla $(M=27.94, D T=4.36)$ se mantiene significativa $(t=-2.215, p<0.0049)$. Sin embargo, la identificación con la ciudad de residencia disminuye de manera estadísticamente significativa si comparamos la primera y la segunda observación $(t=2.776, p<0.007)$.

Cambios en la red social y en las redes personales

Desde el punto de vista de la red completa, el fin de los estudios secundarios inicia un proceso paulatino de disgregación de las relaciones (Maya Jariego et al., 2015). Estudiantes que 
durante años han compartido un mismo espacio institucional, en el Instituto de Enseñanza Secundaria, se separan en diferentes contextos de relación. Una parte inicia sus estudios universitarios, mientras que otros no continúan sus estudios o repiten curso. Por su parte, aquellos que van a la universidad, se disgregan en diferentes facultades, en diferentes campus $\mathrm{o}$ incluso en diferentes instituciones. Este proceso se inicia precisamente entre nuestras dos observaciones. Sin embargo, en este caso la tasa de cambios parece más lenta que la que hemos descrito en las variables que reflejan el nuevo estilo de vida metropolitano.

La red de conocidos aumenta ligeramente en densidad y en la centralidad de grado promedio entre ambas observaciones. Concretamente, la densidad pasa de 0.846 a 0.876 , y el grado promedio (average degree), de 58368 a 60 440. La proporción de datos perdidos en T2 es de 0.32 . No obstante, los cambios que se advierten en la red dependen del tipo de lazo en el que centremos el análisis, como demostramos en la Figura 1. Como se observa en el gráfico, el proceso de disgregación parcial de la red puede quedar más patente poniendo el foco en los lazos fuertes, ya sean vínculos de amistad o personas con las que tienen "bastante relación" (Figura 1). De hecho, la red de lazos débiles (con valores de 1 a 3) aumenta en densidad (de 0.558 a 0.627), mientras que la de lazos fuertes (valores 4 y 5 ) disminuye (de 0.307 a 0.249) en el mismo periodo de observación. En la representación también se aprecia que aquellos que no terminan sus estudios de secundaria ocupan la periferia de la red de amistad y serían posiblemente los primeros en desvincularse de una red completa en proceso de disgregación (Figura 1).
Figura 1

Red de lazos débiles y red de lazos fuertes en T1 y T2.

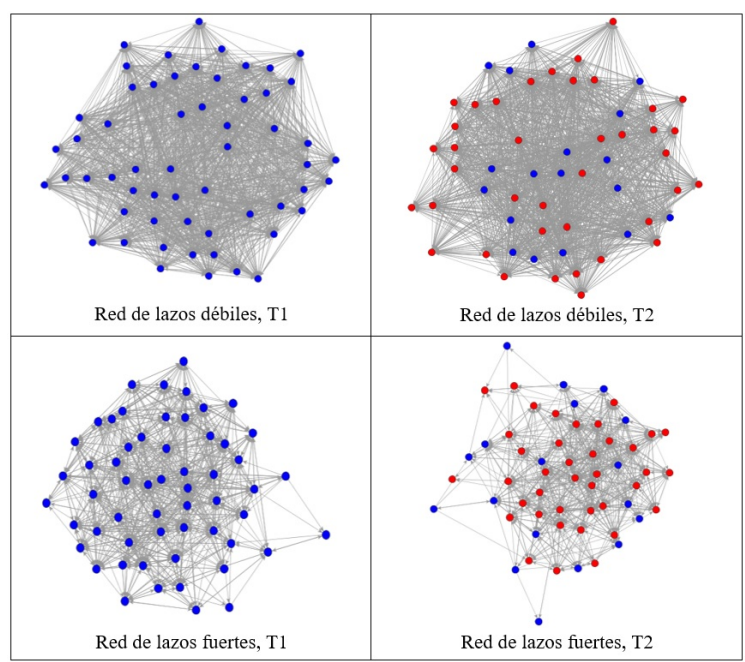

La red de lazos débiles está elaborada con las relaciones en las que "les suena el nombre", se conocen o "hablan de vez en cuando". La red de lazos fuertes corresponde a relaciones en las que "tienen bastante relación" o son amigos. En azul se indican los encuestados que cursan sus estudios en Alcalá y en rojo en Sevilla

Descendiendo al nivel de las redes personales, en líneas generales parece observarse una ligera reducción de la cohesión estructural (Tabla 1). Concretamente, las redes personales tienen un menor grado de centralidad promedio $(t=3.025$, $p<0.004)$ y menor densidad $(t=2.928$, $p<0.005)$, mientras que aumenta de forma significativa el grado de intermediación promedio $(t=-2.181, p<0.033)$. La transición entre dos contextos socio-geográficos parece dar lugar a cambios significativos en la estructura de la red personal, que podrían a su vez predisponer al individuo para su incorporación al nuevo contexto.

\section{Tabla 1}

Prueba t para muestras apareadas: redes personales de estudiantes ingresando en la universidad

\begin{tabular}{|c|c|c|c|c|c|c|}
\hline \multirow{2}{*}{$\begin{array}{c}\text { Redes } \\
\text { personales }\end{array}$} & \multicolumn{2}{|c|}{$\begin{array}{l}\text { Primera oleada (T1) } \\
\qquad N=57\end{array}$} & \multicolumn{2}{|c|}{$\begin{array}{c}\text { Segunda Oleada (T2) } \\
\qquad N=57\end{array}$} & \multirow[t]{2}{*}{$t$} & \multirow{2}{*}{ Sig. } \\
\hline & $M$ & $D T$ & $M$ & DT & & \\
\hline Av. Degree & 41.02 & 11.2 & 36.84 & 9.76 & 3.025 & 0.004 \\
\hline Av. Close & 53.08 & 16.47 & 51.69 & 16.69 & 0.567 & 0.573 \\
\hline & & 0. & & 0.64 & -2.181 & 0.033 \\
\hline Av. Eigenvect & 18.57 & 1.13 & 17.7 & 4.05 & 1.677 & 0.099 \\
\hline Av. Density & 0.64 & 0.19 & 0.58 & 0.16 & 2.928 & 0.005 \\
\hline N. Cliques & 63.68 & 62.67 & 68.35 & 111.21 & -0.524 & 0.603 \\
\hline$N$. Components & 1.47 & 1.23 & 1.35 & 1.14 & 1.187 & 0.24 \\
\hline
\end{tabular}


En un estudio previo, con una muestra transversal de estudiantes universitarios alcalareños, observamos una correlación significativa entre la densidad de sus redes personales y el grado de centralidad promedio $(r=0.187, p<0.05)$, la cercanía promedio $(r$ $=0.183, p<0.05)$ y el eigenvector promedio $(r=0.171, p<0.05)$ (Araya \& Maya Jariego, 2005; Maya Jariego \& Holgado, 2005). Sin embargo, en este caso ninguna de las 56 correlaciones bilaterales entre los indicadores de centralidad de las redes personales y la red social completa resultó significativa, en ninguna de las dos oleadas. Con este antecedente, a continuación comprobamos el efecto de la estructura de las redes personales en sendos modelos longitudinales de la red social.

Análisis de simulación longitudinal de las redes sociales

Finalmente, la información sobre las redes personales se introdujo como co-variantes individuales en RSIENA (Snijders, 2001, 2005). Para ello recurrimos al indicador de centralidad que en los análisis previos comprobamos que tiende a aumentar a lo largo del proceso de transición socio-geográfica: la intermediación promedio.

Confeccionamos un modelo con la red de conocidos, tomando como factores endógenos la densidad, la reciprocidad, la transitividad y la similitud en género, que han mostrado ser dimensiones relevantes en la evolución de las redes sociales de los jóvenes (e.g., Knecht, Snijders, Baerveldt, Steglich, \& Raub, 2010; Lubbers, Snijders, \& Van Der Werf, 2011). Por su parte, como indicador de las redes personales, se incorporó el grado de intermediación promedio (average betweenness) de la red personal observada en la primera oleada. Este indicador, que fue introducido como covariante en el modelo RSIENA, representa la modularidad de la red personal, y lo utilizamos en el modelo para predecir dos tendencias: la probabilidad de formar lazos (y de no romperlos), y la rapidez con la que se cambian los mismos. También evaluamos el impacto de ambas variables de las redes personales de los entrevistados en la tasa de cambio de la red completa.

Tanto en la red de conocidos como en la red de amigos, se observa que la tasa de cambio (es decir, crear lazos no existentes y disolver lazos existentes) es alta. El efecto negativo significativo de la densidad sugiere que la probabilidad de mantener lazos existentes o crear nuevos lazos con personas aleatorias es bajo. Una tendencia, por otro lado, esperable en una cohorte de estudiantes de secundaria en proceso de disgregación. No obstante, tanto para los lazos débiles como para los lazos fuertes, se observa que la incrustación estructural (embeddenness) protege a los lazos contra su ruptura. Si un lazo es recíproco, o si el par tiene relaciones con terceros, es más probable que se mantenga el lazo (o que se forme uno nuevo, si no existía previamente). También observamos que es más fácil que se mantengan los lazos entre los alumnos del mismo género (o que se formen nuevos lazos en el caso de que no existieran en la primera oleada), que entre alumnos de diferentes géneros.

Por su parte, el indicador de intermediación promedio (average betweenness) de la red personal parece influir significativamente en la evolución de la red completa. Los alumnos que tienen redes personales con más intermediación (o indirectamente, redes más modulares) tienen una tasa de cambio más baja que las personas con menos intermediación en sus redes. A la vez, es más probable que se mantengan los lazos débiles, y menos probable que desarrollen nuevos lazos débiles con gente que antes no conocían. Quizás, al estar más acostumbrados a manejar relaciones sociales divididas entre diferentes grupos o ámbitos con pocas relaciones entre sí, son más capaces también de mantener los lazos débiles del instituto. No obstante, es menos probable que mantengan los lazos fuertes en la red de estudiantes, siendo significativamente más probable la ruptura de sus amistades. Es decir, las amistades menos integradas en la red personal parecen más vulnerables y más propensas a desaparecer, como se muestra en la Tabla 2. 
Tabla 2

La red personal como predictor de la evolución de la red socio-céntrica $(n=69)$

\begin{tabular}{|c|c|c|c|c|}
\hline \multirow[t]{2}{*}{ Efectos } & \multicolumn{2}{|c|}{ Red de conocidos } & \multicolumn{2}{|c|}{$\begin{array}{c}\text { Red de relaciones } \\
\text { fuertes }\end{array}$} \\
\hline & AES & SE & AES & SE \\
\hline \multicolumn{5}{|l|}{ Función de cambio } \\
\hline Tasa de cambio (base) & $20.84 * *$ & 1.17 & $22.35^{* *}$ & 1.15 \\
\hline $\begin{array}{l}\text { Efecto intermediación de la red } \\
\text { personal sobre la velocidad }\end{array}$ & $-0.006^{*}$ & 0.002 & 0.006 & 0.003 \\
\hline \multicolumn{5}{|l|}{ Función de evaluación } \\
\hline Grado de salida (densidad) & $-1.85 * *$ & 0.12 & $-1.88 * *$ & 0.06 \\
\hline Reciprocidad & $0.53 * *$ & 0.10 & $0.71 * *$ & 0.08 \\
\hline Transitividad & $0.02 * *$ & 0.001 & $0.07 * *$ & 0.004 \\
\hline Similitud en género & $0.15^{*}$ & 0.06 & $0.30 * *$ & 0.06 \\
\hline \multicolumn{5}{|l|}{ Intermediación red personal } \\
\hline Creación nuevos lazos & $-0.007 * *$ & 0.002 & 0.005 & 0.003 \\
\hline Mantenimiento lazos existentes & $0.01 * *$ & 0.002 & $-0.006^{* *}$ & 0.002 \\
\hline
\end{tabular}

La intermediación promedio es un indicador indirecto del proceso de transición a nivel individual, en este caso, iniciar los estudios universitarios. También puede corresponder a una tendencia personal a manejarse en ámbitos relacionales diferentes, o simplemente al resultado de repartir el tiempo entre círculos sociales distintos.

Con el modelo RSIENA, hemos demostrado que la intermediación promedio de las redes personales parece tener un papel en la evolución y la tasa de cambio de las redes sociales completas de los estudiantes en proceso de reubicación geográfica y/o transición personal. Con análisis complementarios comprobamos que otros indicadores estructurales de las redes personales parecen tener menos influencia y no fueron presentados en los análisis. ¿Qué tipo de interacción se produce entonces entre las propiedades de la red personal y las dinámicas de la red completa?

Dos procesos parecen discurrir en paralelo. Por un lado, compartir la transición normativa se refleja en el mantenimiento de los lazos débiles con conocidos del instituto (mientras que aquellos que siguen en secundaria pueden empezar a desconectarse del resto). Sin embargo, en segundo lugar, la transición normativa también significa abrirse a nuevas relaciones, y con el tiempo podría conducir a incorporar nuevas relaciones de amistad en el espacio personal. Nuevas relaciones que, al menos en sus fases iniciales, estarán menos imbricadas en el conjunto de la red personal. De momento, en el periodo de observación analizado, pudimos comprobar la tendencia significativa entre aquellos con una red personal con mayor intermediación promedio a perder algunos de sus lazos fuertes. Por tanto, parece funcionar como un elemento de predisposición individual en un proceso paulatino de disgregación de la red de compañeros de instituto.

\section{Discusión}

Con esta investigación hemos mostrado que los indicadores de centralidad promedio de las redes personales reflejan indirectamente el proceso de reubicación geográfica a nivel individual. Concretamente, durante la fase más activa de las transiciones ecológicas, las redes personales parecen experimentar un aumento en el grado de intermediación promedio. Por otro lado, la intermediación promedio de las redes personales parece influir también en la evolución y en la tasa de cambio de la red social de la cohorte de estudiantes en proceso de reubicación y/o transición personal. Finalmente, como también discutimos a continuación, la cohorte de estudiantes en proceso de reubicación podría desacelerar los cambios de nivel individual.

Primero, este estudio introduce una innovación al examinar la co-evolución de las redes sociales y las redes personales. Aportamos alguna evidencia empírica que permitiría considerar los indicadores de centralidad de intermediación en la red personal como un proxy indirecto de las transiciones ecológicas en el plano individual. En este caso, utilizamos un indicador genérico de centralidad promedio: average betweenness. Sería de interés reproducir estas mismas observaciones con indicadores de centralización (e.g., betweenness centralization) o de distribución de las relaciones entre los lazos internos y externos al grupo (e.g., E-I index). Pero aún con un indicador grueso de cohesión estructural, se observó una incidencia significativa en la evolución de las relaciones 
en una cohorte de estudiantes en transición ecológica.

Los resultados deben interpretarse con cautela. Entre otras limitaciones, los modelos RSIENA de simulación se aplicaron de modo exploratorio. Solo disponemos de dos observaciones longitudinales -en las que contamos, además, con una pequeña fracción de datos perdidos entre ambas-, y los datos de redes personales se incorporaron sin tratamientos de normalización estadística. Sin embargo, se trata de una de las primeras evidencias de la relación entre propiedades estructurales de la red en el nivel individual y las dinámicas de una red completa. Además, los datos sugieren el papel crítico del grupo de iguales en las transiciones normativas.

En segundo lugar, el grupo de amigos y conocidos con los que se comparte la transición desde la enseñanza secundaria a la enseñanza universitaria (o a otros recorridos personales), es una referencia importante para los cambios que discurren a nivel individual. Es el colectivo que conecta el espacio social de origen, en este caso la ciudad en la que han nacido y vivido toda su vida, con el nuevo contexto institucional y geográfico. La cohorte de estudiantes en proceso de reubicación podría desacelerar las transiciones individuales, al actuar como un amortiguador temporal y proporcionar apoyo. En las transiciones normativas, el individuo no se disuelve aisladamente en un nuevo espacio social, sino que está integrado en un colectivo que lo acompaña a lo largo del proceso de cambio. De hecho, la cohorte de iguales -el grupo de estudiantes que terminan la enseñanza secundaria- conforma la estructura social más inmediata de la que forma parte.

Al terminar la Enseñanza Secundaria, la red de relaciones de los estudiantes de último curso entra en un proceso paulatino de disgregación. En este contexto, la evolución de la red social parece verse influida por dinámicas de desacoplamiento (Grossetti, 2005) y de competencia entre relaciones múltiples activas (Maya Jariego, 2006). Este proceso es más evidente cuando se toma en consideración la fortaleza de la relación en la dinámica de las redes completas (Maya Jariego et al., 2015). No obstante, los modelos exploratorios con la red de conocidos permiten apuntar algunas tendencias que sería interesante documentar en la investigación futura.

Por un lado, el individuo deja de frecuentar un conjunto de relaciones cuya interacción estaba vinculada al contexto institucional de referencia, en este caso el Instituto de Enseñanza Secundaria. Dichas relaciones pasan a estar latentes y ya no forman parte de las interacciones interpersonales del día a día, como ocurre con los contactos activos. Sin embargo, algunas de las relaciones más fuertes, especialmente los vínculos de amistad, se desacoplan, es decir, se mantienen con independencia del contexto institucional en el que se originaron y acompañan al individuo en una nueva etapa de su ciclo vital.

Por otro lado, al iniciar los estudios en una ciudad diferente a la localidad de residencia, la actividad del individuo se distribuye entre dos espacios socio-geográficos diferenciados. De algún modo, las relaciones previas compiten con las nuevas. Cada persona tiene una capacidad limitada para el inicio y el mantenimiento de relaciones. De forma que el desarrollo de relaciones de compañerismo y amistad en un nuevo espacio social-como puede ser el contexto universitario- introduce nuevas relaciones, que no están incrustadas con las anteriores y transforman, al menos temporalmente, la estructura de la red personal.

Como los resultados de este trabajo parecen ilustrar, un descenso en el grado de cohesión de las redes personales podría ser un indicio de transición ecológica. Los estudiantes que se inician en un patrón de movilidad metropolitana (de ida y vuelta), y distribuyen su tiempo entre dos localidades, experimentan un aumento en el grado de intermediación en sus redes personales. A su vez, este tipo de estructuras están mejor predispuestas a la incorporación de nuevas relaciones. De ahí que la distribución colectiva de las redes personales con mayor intermediación promedio podría informar indirectamente de la labilidad relacional en una cohorte determinada. Es decir, una mayor prevalencia de individuos en transición, por lo general más abiertos a 
la formación de nuevas relaciones, da lugar a comunidades con una mayor tasa de cambio relacional. Es lo que ocurre por ejemplo en el caso de los estudiantes Erasmus (Cachia, 2014; De Federico, 2003).

Tanto las dinámicas de desacoplamiento como la competencia entre relaciones múltiples activas hacen necesario tener en cuenta en futuros estudios el tipo de relación y la fortaleza del lazo. Es probable que la mayor proporción de cambios en las transiciones ecológicas tengan lugar en la periferia de la red, con la substitución de unos lazos débiles por otros. Sin embargo, las observaciones de este trabajo nos llevan a pensar que los cambios, proporcionalmente menos numerosos, en las relaciones con un valor psicológico moderado y alto tienen una repercusión de mayor alcance en la estructura de las redes personales y las redes completas.

\section{Agradecimientos}

El trabajo de campo se realizó con el apoyo de las Acciones Complementarias del Ministerio de Educación y Ciencia (SEJ2005-25683) y el Ayuntamiento de Alcalá de Guadaíra (Sevilla). La investigación formó parte del proyecto "Dynamics of actors and networks across levels: individuals, groups, organizations and social settings" (2006-2009) de la European Science Foundation (ESF). Una versión preliminar de los análisis de simulación de redes se presentó en el "European Research Collaborative Project Meeting on Dynamic Analysis of Networks and Behaviours", celebrado en el Nuffield College de la Universidad de Oxford, el 30 de junio de 2009. El trabajo ha tenido continuidad en el proyecto "Social Influence in Dynamic Networks" (2012-2014), European Collaborative Research Project (ECRP), y European Science Foundation (10-ECRP-044). Agradecemos a los profesores Juan Antonio Muñoz y Javier Hermida las facilidades ofrecidas para acceder a los estudiantes del IES Albero, así como la colaboración de todos los participantes en el estudio.

\section{Referencias}

Araya, R., \& Maya Jariego, I. (2005). Los Puentes Interlocales: las Redes Personales de los Universitarios Alcalareños en Sevilla. En J. I. Porras, \& V. Espinoza (Eds.), Redes: Enfoques y Aplicaciones del Análisis de Redes Sociales (pp. 183-213). Santiago de Chile: Universidad Bolivariana. ISBN: 9568024271

Arnett, J. J. (2000). Emerging adulthood: A theory of development from the late teens through the twenties. American Psychologist, 55(5), 469-480. http://doi.org/10.1037//00 03-066X.55.5.469

Barrera, M. (1980). A method for the assessment of social support networks in community survey research. Connections, 3(3), 8-13. Recuperado de http://insna.org/PDF/Conn ections/v3/1980_I-3.pdf

Borgatti, S. P., Everett, M. G., \& Freeman, L. C. (2002). Ucinet for Windows: Software for social network analysis. Harvard, MA: Analytic Technologies.

Brandes, U., \& Wagner, D. (2004). Analysis and Visualization of Social Networks. En M. Jünger, \& P. Mutzel (Eds.), Graph Drawing Software (pp. 321-340). Nueva York, NY: Springer-Verlag. https://doi.org/10.1007/97 8-3-642-18638-7_15

Bronfenbrenner, U. (1977). Toward an experimental ecology of human development. American Psychologist, 32(7), 513-531. https://doi.org/10.1037//0003-06 $6 x .32 .7 .513$

Cachia, R. (2014). Connected Lives in Contemporary Mobile Societies (Tesis doctoral). Sevilla: Universidad de Sevilla. Recuperado de https://idus.us.es/xmlui/han dle/11441/61250

Chavis, D. M., Hogge, J. H., McMillan, D. W., \& Wandersman, A. (1986). Sense of community through Brunswik's lens: A first look. Journal of community psychology, 14(1), 24-40. https://doi.org/10.1002/1520-6629( 198601)14:1\%3C24::aid-jcop2290140104 \%3E3.0.co;2-p 
Connell, J., \& Furman, W. (1984). The study of transitions. En R. Emde, \& R. Harmon (Eds.), Continuities and discontinuities in development (pp. 153-173). Nueva York, NY: Plenum Press. ISBN: 978-1-4613-2725-7

Domínguez, S., \& Maya Jariego, I. (2008). Acculturation of host individuals: Immigrants and personal networks. American Journal of Community Psychology, 42(3-4), 309-327. https://doi.org/10.1007/s 10464-008-9209-5

de Federico, A. (2003). La dinámica de las redes de amistad. La elección de amigos en el programa Erasmus. Redes: Revista hispana para el análisis de redes sociales, 4(3). https:/ /doi.org/10.5565/rev/redes.42

de Federico, A. (2005). El análisis dinámico de redes sociales con SIENA. Método, discusión y aplicación. Empiria. Revista de metodología de ciencias sociales, 10, 151-184. http://doi.org/10.5944/empiria.10 .2005 .1047

Freeman, L. (1977). A set of measures of centrality based upon betweenness. Sociometry, 40(1), 35-41. https://doi.org/10 $.2307 / 3033543$

Gómez-Jacinto, L., \& Hombrados-Mendieta, I. (1992). Sentido de comunidad y privacidad. Revista de Psicología Social, 7(2), 213-226. https://doi.org/10.1080/02134748 .1992 .10821662

Grossetti, M. (2005). Where do social relations come from? A study of personal networks in the Toulouse area of France. Social networks, 27(4), 289-300. https://doi.org/10.1016/j.so cnet.2004.11.004

Knecht, A., Snijders, T. A. B., Baerveldt, C., Steglich, C. E. G., \& Raub, W. (2010). Friendship and Delinquency: Selection and Influence Processes in Early Adolescence. Social Development, 19(3), 494-514. https:// doi.org/10.1111/j.1467-9507.2009.00564.x

Lubbers, M. J., Snijders, T. A. B., \& Van Der Werf, M. P. C. (2011). Dynamics of Peer Relationships Across the First Two Years of Junior High as a Function of Gender and Changes in Classroom Composition.
Journal of Research on Adolescence, 21(2), 488-504. https://doi.org/10.1111/j.1532-77 95.2010.00688.x

Maya Jariego, I. (1999). Análisis de los recursos de apoyo social de los inmigrantes africanos y latinoamericanos en Andalucía. Tipología de redes y proceso de adaptación (Tesis doctoral). Sevilla: Universidad de Sevilla. Recuperado de https://idus.us.es/xmlui/handle/11441/1 5506

Maya Jariego, I. (2004). Sentido de comunidad y potenciación comunitaria. Apuntes de psicología, 22(2), 187-211. Recuperado de http://www.apuntesdepsicologia.es/inde x.php/revista/article/download/50/52

Maya Jariego, I. (2006). Webs of compatriots: Relationship networks among immigrants. En J. L. Pérez-Pont (Ed.), Geografías del desorden. Migración, alteridad y nueva esfera social (pp. 257-276). Valencia: Universidad de Valencia. ISBN: 9788437065564

Maya Jariego, I., \& Armitage, N. (2007). Multiple Senses of Community in Migration and Commuting: The Interplay between Time, Space and Relations. International Sociology, 22 (6), 743-766. http s://doi.org/10.1177/0268580907082259

Maya Jariego, I., \& de Federico A. (2006). El Análisis Dinámico de Redes con Siena. En J. L. Molina, A. Quiroga, J. Martí, I. Maya Jariego, \& A. De Federico (Eds.), Talleres de Autoformación con Programas Informáticos de Análisis de Redes Sociales (pp. 90-115). Barcelona: Universitat Autònoma de Barcelona. Recuperado de http://person al.us.es/isidromj/php/wp-content/uploads/2 007/07/el-analisis-dinamico-de-redes-con-s iena.pdf

Maya Jariego, I., \& Holgado, D. (2015). Living in the metropolitan area. Correlation of interurban mobility with the structural cohesion of personal networks and the originative sense of community. Psychosocial Intervention, 24(3), 185-190. https://doi.org /10.1016/j.psi.2015.09.001

Maya Jariego, I., \& Holgado, D. (2005). Lazos fuertes y proveedores múltiples de apoyo: comparación de dos formas 
de representación gráfica de las redes personales. Empiria. Revista de metodología de ciencias sociales, (10), 107-128. https://d oi.org/10.5944/empiria.10.2005.1045

Maya Jariego, I., Lubbers, M., \& Molina, J. L. (2015). The dynamics of a secondary school cohort over the transition to university: a friendship network in decay. Submitted for publication.

McCarty, C. (2002). Structure in personal networks. Journal of social structure, 3(1). Recuperado de https:/www.cmu.edu/joss/c ontent/articles/volume3/McCarty.html

McMillan, D. W., \& Chavis, D. M. (1986). Sense of community: A definition and theory. Journal of community psychology, 14(1), 6-23. https://doi.org/10.1002/1520-6629(1 98601) 14:1\%3C6::aid-jcop2290140103\%3 E3.0.co;2-i

Ruble, D. N., \& Seidman, E. (1996). Social transition: Windows into social psychological transactions. En E. T. Higgins, \& A. W. Kruglanski (Eds.), Social psychology: Handbook of basic principles (pp. 830-856). Nueva York, NY: Guildford Press. ISBN: 9781572309180

Sánchez-Vidal, A. S. (2001). Medida y estructura interna del sentimiento de comunidad: un estudio empírico. Revista de Psicología Social, 16(2), 157-175. https://doi.org/10.1174/02 1347401317351116

Seidman, E., \& French, S. E. (2004). Developmental trajectories and ecological transitions: A two-step procedure to aid in the choice of prevention and promotion interventions. Development and Psychopathology, 16(4), 1141-1159. https:// doi.org/10.1017/s0954579404040179

Snijders, T. A. (2001). The statistical Evaluation of Social Network Dynamics. En M. E. Sobel, \& M. P. Becker (Eds.), Sociological Methodology (pp. 361-395). Londres: Basil Blackwell. ISBN: 9781506323527

Snijders, T. A. (2005). Models for Longitudinal Network Data. En P. Carrington, J. Scott, \& S. Wasserman (Eds.), Models and methods in social network analysis (pp. 215-247). Nueva
York: Cambridge University Press. https://d oi.org/10.1017/cbo9780511811395.011

Valera, S. (1997). Estudio de la relación entre el espacio simbólico urbano y los procesos de identidad social. Revista de Psicología Social, 12 (1), 17-30. https://doi.org/10.1174/0213 47497320892009

\section{Notas}

* Artículo de investigación. Estudio financiado en el marco del proyecto "Dynamics of actors and networks across levels: individuals, groups, organizations and social settings" (2006-2009) de la European Science Foundation (ESF), con las Acciones Complementarias del Ministerio de Educación y Ciencia de España (SEJ2005-25683). 